\title{
Strain Hardening of Carbon Steel During Wire Drawing
}

\author{
Umberto Prisco $^{a}$ * \\ ${ }^{a}$ Dipartimento di Ingegneria Chimica, dei Materiali e della Produzione Industriale, Università degli \\ Studi di Napoli Federico II, Piazzale Vincenzo Tecchio, 80, 80125 Napoli, Italia
}

Received: March 21, 2017; Revised: December 11, 2017; Accepted: February 07, 2018

The Avitzur's limit analysis for the calculation of the drawing force in a wire drawing process is used to study the real behaviour of a carbon steel in an industrial case. The linear hardening assumption, used by Avitzur only for the first step, is extended to the whole process and tested against experimental data. The result shows that the predictions resulting from this extension are in good agreement with the experimental results and are, as rule, conservative.

Keywords: wire drawing, strain-hardening, Avitzur's limit analysis, carbon steel.

\section{Introduction}

Drawing is a cold metal forming technique widely used to obtain rods, pipes, and wires. It is used to manufacture parts with a fine microstructure which improves the mechanical properties of the product, such as its tensile strength. It is very useful to obtain products with small section.

The drawing force is a fundamental parameter for the optimization of the process and of the production costs. Various analytical models have been proposed for the foreseeing of the drawing force, but the one proposed by Avitzur, based on the limit analysis, is very effective because it accounts for the strain hardening effects on the material. In this paper the hypothesis of strain hardening used by Avitzur for the calculation of force in wire drawing is compared with the real behaviour of a carbon steel subjected to an industrial drawing process.

Fig. 1 show a sketch of the die and of the workpiece. In regard to the model adopted in this paper, the factors involved during the process are:

1. reduction area; it can be evaluated with the $R_{i} / R_{f}$ ratio between entrance and exit radius, or using the ratios $\left(R_{i} / R_{f}\right) 2$ and $\left(A_{i}-A_{f}\right) / A_{i}$;

2. semiconical angle of the die, $\alpha$;

3. shear factor, $m$;

4. coefficient of friction, $\mu$;

5. yield limit at uniaxial load, $\sigma_{0}$;

6. wire stress at the exit of the die, $\sigma_{x f}$;

7. wire stress at the entrance of the die, $\sigma_{x b}$;

8. length of the land of the die, $L$;

9. initial and final radius of the wire, $R_{i}$ and $R_{f}$;

10. entrance and exit velocity of the wire, $v_{i}$ and $v_{f}$, respectively.

Plastic work and friction at the workpiece-die interface generate the heat in cold wire drawing. The heat generated induces a temperature gradient, in both the workpiece and

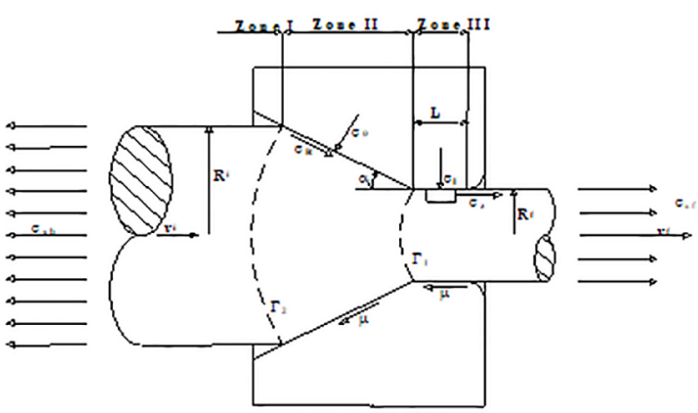

Figure 1. Geometry of the rod and the die

the die, which influences thermal expansion, deformation pattern, and elastic recovery. The determination of the two shares of the drawing force, the one due to the plastic work and the one due to the friction, is very important to build predictive models of the drawing process. In particular, the forecast of the plastic work plays a fundamental role in consideration of the fact that the drawn material experiences work hardening along the various steps of the wire drawing, so that its yield strength is not constant.

The most interesting results, with regard to the calculation of drawing force, were obtained by Avitzur ${ }^{1,2}$, even if Siebel and $\mathrm{Sach}^{3}$ offered solutions still used in practice and in some theoretical formulation due to their easy implementation. On the other hand, the problem of the friction in wire drawing was addressed, among the others researchers, by Moon and $\mathrm{Kim}^{4}$ who, using procedures of inverse engineering, determined the friction and thermal conditions by comparing easy-to-measure variables with the computational results of the drawing power and the temperature distribution of the die. Dixit et al..$^{5}$ studied the effect of the coefficient of friction at the interface on the quality of the drawn product and observed that a decrease in the coefficient of friction tends to make the product more homogeneous and consequently of higher quality. By measuring the temperature in the 
deformation zone and the drawing force by changing the drawing velocity, Vega et al. ${ }^{6}$ confirmed from their experiment that the temperature and the drawing force were a function of the interface conditions. To calculate the friction coefficient many method had been proposed: for example, $\mathrm{Leu}^{7}$ used a model of plastic hemispherical contact against a rigid flat and described the deformed shape as the fractional profile of an ellipsoid, while Ajiboyeet al. ${ }^{8}$ performed an ANOVA analysis comparing two regression models to predict the shear friction factor at the punch interface. Similar models and experimental analyses are presented by Parida et al. ${ }^{17,18}$, Souza et al. ${ }^{13}$ and Andrade et al. ${ }^{14}$.

The principal aim of this paper is to compare the hypothesis of strain hardening used by Avitzur in the model proposed in ${ }^{1}$ for the calculation of the force in wire drawing with the real behaviour of a carbon steel subjected to an industrial drawing process. In particular, the feasibility of the extension of the linear hardening hypothesis, used by Avitzur only in the first step of the wire drawing, to the whole process is analysed and tested against experimental data. This study can provide a simple and useful model to predict the drawing force and power in a multi-pass wire drawing process.

\subsection{Calculation procedures}

Avitzur used an approach that produced two solutions: the upper-bound solution gives, in regard of the predicted drawing power, a value greater or at least equal to the real one; the lower bound solution gives a value lower or at least equal to the real one.

Fig. 2 shows the cinematic field used in Avitzur's theory. He defined the cinematic field as spherical and considered the die as a rigid body. By choosing a kinematic field congruent with the boundary conditions, he was able to calculate the force needed for the drawing process. Two different models are adopted for the study of the friction ${ }^{9,10}$. The first one uses a constant shear factor; the second one is the classical Coulomb law. The present work makes reference only to the first model. Strain hardening modelling is fundamentalin the precise calculation of the drawing force. Avitzur's method assumes that the linear strain hardening follow the function:

$$
\sigma=S(1+\beta \varphi)
$$

Where $S$ is Avitzur initial flow stress, $\beta$ is the strain hardening coefficient and $\varphi$ is the instantaneous effective strain expressed by:

$$
\varphi=-2 \frac{\left(1-\frac{11}{12} \sin ^{2} \theta\right)^{\frac{1}{2}}}{\cos \theta} \int \frac{d r}{r}
$$

where $\theta$ is the angle of the deforming fibre, see Fig. 2 . The solution of the integral yields the expression:

$$
\varphi=2 \frac{\left(1-\frac{11}{12} \sin ^{2} \theta\right)^{\frac{1}{2}}}{\cos \theta} \ln \frac{r_{0}}{r}
$$

Under the hypothesis of linear strain hardening, the upper bound solution has the expression:

$$
\sigma_{x f}=\sigma_{x b}+A
$$

where $A$ is given by:

$$
\begin{gathered}
A=S\left\{\begin{array}{l}
2 f(\alpha) \ln \frac{R_{i}}{R_{f}}+\frac{2}{\sqrt{3}}\left(\frac{\alpha}{\sin ^{2} \alpha}-\cot \alpha\right)+ \\
m(\cot \alpha) \ln \frac{R_{i}}{R_{f}}+m \frac{L}{R_{f}}
\end{array}\right\}+ \\
\beta\left\{\begin{array}{l}
\frac{11}{6}\left[1-\frac{2}{11} \frac{\ln (\cos \alpha}{\sin \alpha}\right]\left(\ln \frac{R_{i}}{R_{f}}\right)^{2}+\frac{4}{\sqrt{3}} F(\alpha) \\
\ln \frac{R_{i}}{R_{f}}+\frac{2}{\sqrt{3}} m \frac{\left(1-\frac{11}{12} \sin ^{2} \alpha\right)^{\frac{1}{2}}}{\sin \alpha} \\
\left(\ln \frac{R_{i}}{R_{f}}\right)^{2}+\frac{4}{\sqrt{3}} m \frac{L}{R_{f}} \frac{\left(1-\frac{11}{12} \sin ^{2} \alpha\right)^{\frac{1}{2}}}{\cos \alpha} \ln \frac{R_{i}}{R_{f}}
\end{array}\right\}
\end{gathered}
$$

In the expression (5) $F(a)$ is represented by:

$$
F(\alpha)=\frac{E(\alpha)}{\sin ^{2} \alpha}
$$

while $E(a)$ is given by:

$$
\begin{aligned}
& E(\alpha)=\frac{5}{\sqrt{11 \cdot 12}} \sin ^{-1}\left(\left(\frac{11}{12}\right)^{\frac{1}{2}} \sin \alpha\right)-\frac{\sin \alpha}{2} \\
& \left(1-\frac{11}{12} \sin ^{2} \alpha\right)^{\frac{1}{2}}+\frac{1}{\sqrt{12}} \ln \frac{\left(1-\frac{11}{12} \sin ^{2} \alpha\right)^{\frac{1}{2}}+\frac{\sin \alpha}{\sqrt{12}}}{\cos \alpha}
\end{aligned}
$$

Eq. (1) states that the central fibre (characterized by the angle $\theta$ equal to 0 ) has the following material characteristic curve:

$$
\sigma=S\left(1+2 \beta \ln \frac{r_{0}}{r}\right)
$$

Eq. (8) can also be written as :

$$
\sigma=S\left(1+\beta \ln \frac{A_{0}}{A}\right.
$$

The die semi-angle commonly employed in industrial practice are rather small, thus the strain-hardening effects in peripheral zone can be considered equal of those present in the core.

\subsection{Experiments}

A series of tensile tests for a 0.8 carbon steel wire in various steps of a complete drawing process were carried out, according to the industrial practise ${ }^{13,14,15}$. From the results of the tests it was possible to evaluate deformation and strain hardening effects on the mechanical characteristics of materials ${ }^{11,12,16}$. The drawing bench, where the wire was drawn, consists of ten steps. The initial diameter of the rod 


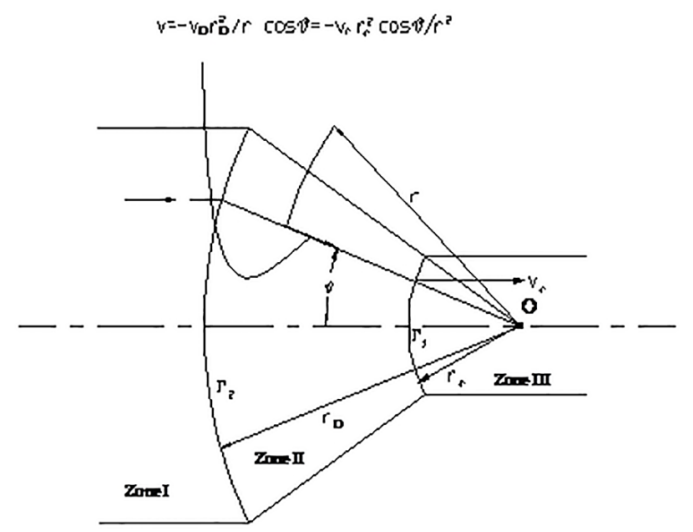

Figure 2. Admissible cinematic field

is $7.00 \mathrm{~mm}$ and the final one is $2.26 \mathrm{~mm}$, each die having at their end a cylindrical zone $1 / 3$ of final diameter long.

The die semi-angle is $5^{\circ}$. Table 1 reports the entrance and the exit diameter for each of the ten steps. Table 2 reports the yield strength after each step.

Table 1. Entrance and exit diameter at each of the ten drawing steps.

\begin{tabular}{lcc}
\hline & Entrance Diameter $(\mathrm{mm})$ & Exit diameter $(\mathrm{mm})$ \\
\hline Step I & 7.00 & 6.04 \\
Step II & 6.04 & 5.25 \\
Step III & 5.25 & 4.58 \\
Step IV & 4.58 & 4.01 \\
Step V & 4.01 & 3.54 \\
Step VI & 3.54 & 3.13 \\
Step VII & 3.13 & 2.79 \\
Step VIII & 2.79 & 2.50 \\
Step IX & 2.50 & 2.36 \\
Step X & 2.36 & 2.26 \\
\hline
\end{tabular}

Table 2. Yield strength after each step.

\begin{tabular}{|c|c|c|c|c|c|c|c|}
\hline $\operatorname{Ln}\left(A_{0} / A\right)$ & 0.0 & 0.148 & 0.424 & 0.557 & 1.030 & 1.087 & 1.131 \\
\hline $\begin{array}{l}\text { Yield } \\
\text { strength } \\
(\mathrm{MPa})\end{array}$ & 630 & 900 & 1150 & 1250 & 1590 & 1630 & 1680 \\
\hline
\end{tabular}

The rod material is a carbon steel $(0.8 \% \mathrm{C})$, its raw tensile stress is $630 \mathrm{Mpa}$. After the drawing to the final diameter of $2.26 \mathrm{~mm}$, its measured tensile stress is $1600 \mathrm{MPa}$.

The preliminary operations, to which the rods are subject before the drawing process, are:

a. pickling in hydrochloric acid;

b. treatment using Bonder salt in hot water;

c. phosphatization at $80^{\circ} \mathrm{C}$;

d. dipping in a borax solution.

A zinc triphosphate layer is applied to guarantee a high lubricant adhesion. The first two dies of the process use calcium stearate as dry lubricant while the other eight use sodium stearate. The solid lubricants ensure boundary lubrication. The calcium stearate guarantees a good protection in spite of the oxide layer always present on the rod; the sodium stearate is more resistant at the high temperatures found during the later stages of the process.

\section{Results and Discussion}

Fig. 3 shows the $\sigma-\varepsilon$ diagram for the starting $(7.00 \mathrm{~mm}$ diameter) and the final (2.26 final diameter) geometry. It is evident that the strain-hardening caused by the drawing double the yield limit of the wire.

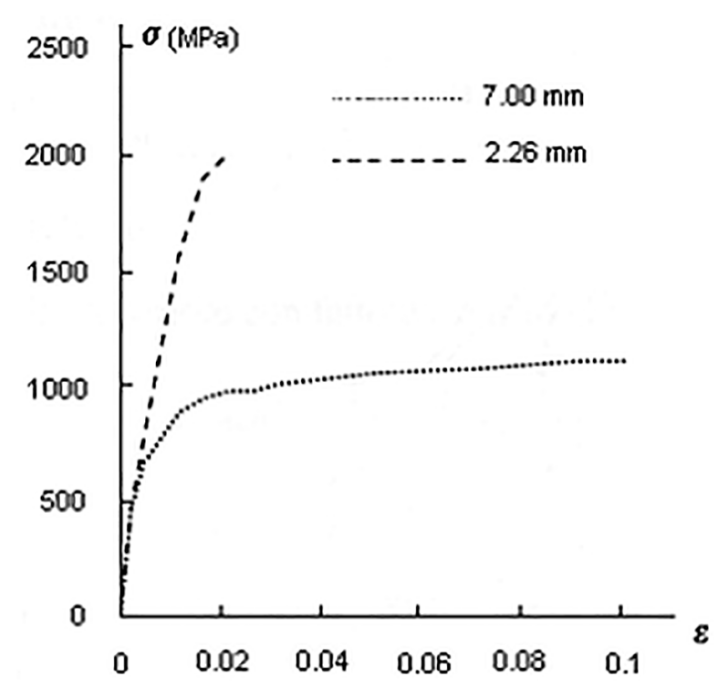

Figure 3. $\sigma-\varepsilon$ diagram for $7 \mathrm{~mm}$ and $2.26 \mathrm{~mm}$ wire diameter

Fig. 4 reports the correlation, after six steps, between the yield limit at the end of each drawing step and the ratio between the actual and the starting area of the wire. Each point is the average over ten measures taken for each single step. When plotted on a semi-logarithmical scale the experimental points can be approximated with a straight line.

Using a linear fitting, it is possible to obtain the equation that best fits the experimental results:

$$
\sigma=630(1+1.52) \ln \frac{A_{0}}{A}
$$

The correlation coefficient of the adopted regression model (a measure of the capacity of the regression to fit the phenomenon, it has a limit value equal to 1 ) is 0.978 . This result permits to state that the linear strain hardening hypothesis, adopted by Avitzur for a single step, is extensible to a multi-step drawing.

Further, the linear strain hardening parameters, $S$ and $\beta$, are constant within all the steps of the examined drawing process, and they are equal to those found for the first step. 


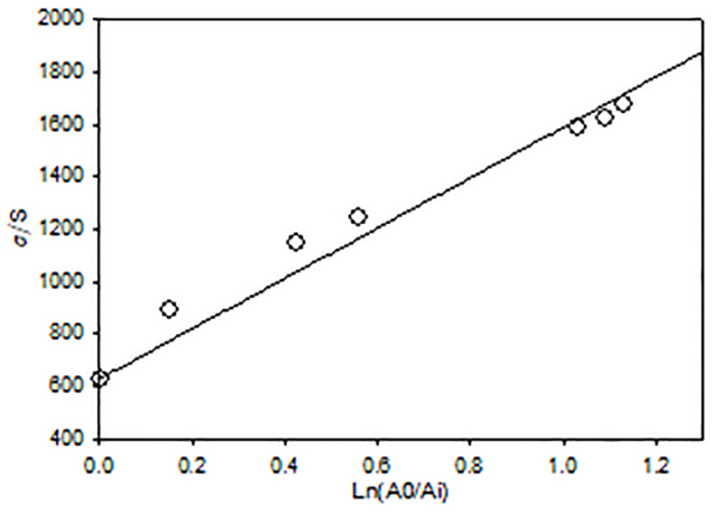

Figure 4. Experimental results relating $\sigma / S$ to $\operatorname{Ln}\left(A_{0} / \mathrm{A}_{\mathrm{i}}\right)$

Starting from this assumption, it is possible to evaluate the material characteristic parameters $S$ and $\beta$ needed to calculate the drawing force for each step. The knowledge of the drawing force and of the strain hardening properties allows the calculation of the friction coefficient. In this way, all process parameters can be calculated; in our condition, for example, the computed shear factor calculated using the Avitzur model is $m=0.11^{12,19}$.

In Fig. 5 the predicted drawing stress for a 0.8 carbon steel with a reduction area of $25 \%$ in function of the semiangle $\alpha$ is shown (friction factor $m=0.11$ and length of the die equal to $1 / 3$ of the final diameter) Fig. 6 show the drawing stress for the same material with $m=0.11$ and $\alpha=5^{\circ}$ in function of the reduction area. In both casesAvitzur's formulation for strain hardening materials was used together with a second formulation that utilizes as yield limit an average value obtained using for each step the entrance and final tensile strength. The difference between the two is no more than $3 \%$.

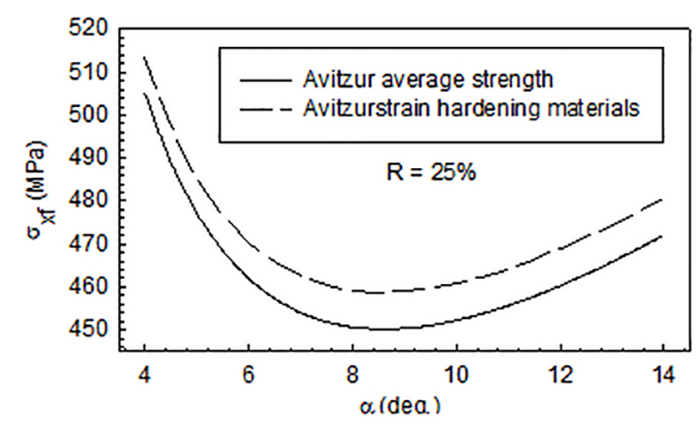

Figure 5. Drawing stress $\sigma_{x f}$ for a reduction area of $25 \%$ and $m=0.11$

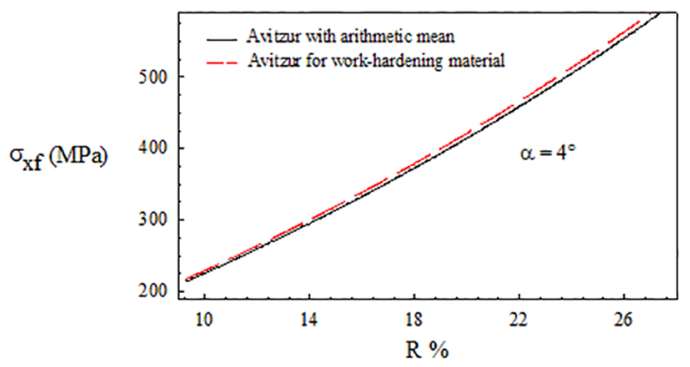

Figure 6. Drawing stress $\sigma_{x f}$ for a $5^{\circ}$ die semiangle and $m=0.11$

\section{Conclusions}

The experimental tests carried out were focused on evaluating if the model adopted by Avitzur for the strain hardening effects is useful in the industrial practice of wire drawing. The experimental data obtained are in good agreement with the theoretical model. It is proven that the characteristic parameters of the raw material can be utilized not only for a single step but also for the entire multi-step process. Indeed, the values of yield strength after each step vs. the logarithm of ratio between actual area and the starting area of wire converge to a straight line. As consequence of these tests it is shown the feasibility to predict the behaviour of an industrial multi-step process by testing the same process parameters in an experimental single-step setup and then extending the obtained results to the whole multi-step process. This should allow to reduce both the amount of test trials on the production line and to avoid teething troubles when some changes in the process are introduced.

\section{References}

1. Avitzur B. Strain-Hardening and Strain-Rate Effect in Plastic Flow Through Conical Converging Dies. Journal of Engineering for Industry. 1967;89(3):556-562.

2. Avitzur B. Analysis of Wire Drawing and Extrusion Through Conical Dies of Small Cone Angle. Journal of Engineering for Industry. 1963;85(1):89-95.

3. Lange K, ed. Handbook of Metal Forming. New York: McGrawHill; 1985.

4. Moon C, Kim N. Analysis of wire-drawing process with friction and thermal conditions obtained by inverse engineering. Journal of Mechanical Science and Technology. 2012;26(9):2903-2911.

5. Dixit US, Dixit PM. An analysis of the steady-state wire drawing of strain-hardening materials. Journal of Materials Processing Technology. 1995;47(3-4):201-229.

6. Vega G, Haddi A, Imad A. Temperature effects on wiredrawing process: experimental investigation. International Journal of Material Forming. 2009;2(Suppl 1):229-232.

7. Leu DK. Evaluation of friction coefficient using simplified deformation model of plastic hemispherical contact with a rigid flat. Journal of Mechanical Science and Technology. 2010;24(8):1697-1707.

8. Ajiboye JS, Jung KH, Im YT. Sensitivity study of frictional behavior by dimensional analysis in cold forging. Journal of Mechanical Science and Technology.2010;24:115-118.

9. Bowden FP, Tabor D. The Friction and Lubrication of Solids. Oxford: Clarendon Press; 1954.

10. Avitzur B. The upper bound approach to the friction wave model. Journal of Materials Processing Technology. 1992;34(14):201-209.

11. Castro ALR, Campos HB, Cetlin PR. Influence of die semiangle on mechanical properties of single and multiple pass drawn copper. Journal of Materials Processing Technology. 1996;60(14):179-182. 
12. Snidle RW. Contribution to the theory of frictional heating and the distribution of temperature in wires and strips during drawing. Wear. 1977;44(2):279-294.

13. Souza TF, Soares CAT, Zottis J, Nunes RM, Rocha AS, Hirsch T. Comparison between Neutron Diffraction measurements and numerical simulation of residual stresses of a Wire-Drawing process. Materials Research. 2013;16(2):508-514.

14. Andrade EC, Bernardi HH, Otubo J. microstructural evaluation on shape recovery in stainless Fe-Mn-Si-Cr-Ni-Co SMA processed by wire drawing. Materials Research. 2014;17(3):583-587.

15. Prisco U. Size-dependent distributions of particle velocity and temperature at impact in the cold-gas dynamic-spray process. Journal of Materials Processing Technology. 2015;216:302314.
16. Kabayama LK, Taguchi SP, Martínez GAS. The influence of die geometry on stress distribution by experimental and FEM simulation on electrolytic copper wiredrawing. Materials Research. 2009;12(3):281-285.

17. Parida AK, Soren S, Jha RN, Sadhukhan S. 12 mm Thick Circular Blanks of Al-killed AISI 1020 Steel -Applied for Cylindrical Cup Manufacturing by Multistage Deep Drawing with Simultaneous Ironing. Materials Research. 2016;19(5):965-976.

18. Parida AK, Soren S, Jha RN, Sadhukhan S, Krishnamurthy N. Multistage Deep Drawing with Ironing of Al-killed AISI 1040 Graded Medium Carbon Steel: a Parametric Study. Materials Research. 2017;20(4):1111-1120.

19. Serroni G, Squillace A, Prisco U, Bitondo C, Prisco A. Pannelli aeronautici rinforzati con parti estruse saldate mediante FSW: caratterizzazione meccanica. La Metallurgia Italiana. 2011;103(1):35-39. 